\title{
Medida da satisfação em relacionamento de casal
}

\author{
João Fernando Rech Wachelke $e^{1}$ \\ Alexsandro Luiz de Andrade \\ Roberto Moraes Cruz 2 \\ Robson Brino Faggiani \\ Jean Carlos Natividade
}

\begin{abstract}
Resumo
O objetivo deste estudo é descrever a construção e validação de uma escala de satisfação em relacionamento de casal, composta por subescalas capazes de medir aspectos componentes da satisfação com relacionamento. Para constituir a Escala Fatorial da Satisfação em Relacionamento de Casal foram elaborados nove itens, no formato escala de Likert de 5 pontos, divididos em dois fatores após a realização da análise dos componentes principais e análise fatorial, Satisfação com Atração Física e Sexualidade e Satisfação com Afinidades de Interesses e Comportamentos, formados por cinco e quatro itens, respectivamente. Os índices de confiabilidade alfa de Cronbach obtidos, 0,76 e 0,61, foram considerados satisfatórios. Os resultados apontam que a satisfação com as afinidades entre os companheiros de relacionamento é menor e mais variável, enquanto a satisfação com a sexualidade e aparência dos companheiros é maior e apresenta medidas mais parecidas entre si.

Palavras-Chave: Relacionamento de casal; Comportamento de afinidade; Satisfação no relacionamento.
\end{abstract}

\section{Measure of the satisfaction in couple relationship}

\begin{abstract}
This study aimed to describe the construction and validation of a couple relationship satisfaction scale, composed by sub-scales for measuring component aspects of relationship satisfaction. For the constitution of the Factorial Scale of Satisfaction in Couple Relationships nine items were developed, in the 5-point Likert scale format, divided in two factors after the principal component analysis and factor analysis, that is, Satisfaction with Physical Attraction and Sexuality and Satisfaction with Affinities of Interests and Behavior, formed by five and four items respectively. The Cronbach alpha reliability indexes, 0,76 and 0,61 , were considered satisfactory. The results suggest that satisfaction with affinities between relationship companions is low and variable, while satisfaction with sexuality and companions' appearance is high and presents more similar measures.

Keywords: Couple relationship; Affinity behavior; Relationship satisfaction.
\end{abstract}

A satisfação em relacionamentos de casal insere-se no contexto dos estudos sobre a qualidade dos relacionamentos amorosos. Há diferentes maneiras de conceituar aspectos qualitativos dos relacionamentos, o que suscita o surgimento de diversas abordagens no que diz respeito às formas de mensurar e relacionar os construtos referidos (Fletcher, Simpson \& Thomas, 2000).

No domínio deste estudo, cabe, inicialmente, definir os construtos satisfação, relacionamento de casal e qualidade de relacionamento. De acordo com o Dicionário Aurélio Básico da Língua Portuguesa (Ferreira, 1988), satisfazer é "agradar, contentar; corresponder ao que se deseja" (p. 588) e satisfeito significa "saciado, contente" (p. 588). Neste trabalho, satisfação será tratada como uma avaliação cognitiva positiva de um objeto, obtida após comparação com objetos semelhantes que possuam características consideradas aceitáveis ou boas. Nesse sentido, a satisfação é considerada um construto voltado fundamentalmente para o nível de análise do indivíduo. Essa definição não entra em contradição com a noção de uma determinação social das características dos objetos em questão ou com a constituição social dos indivíduos, apenas se refere a uma ênfase maior nas especificidades das relações destes com os objetos com os quais estão satisfeitos ou não, dada a multiplicidade de formas que essas relações podem assumir.

Por relacionamento de casal é entendido todo relacionamento amoroso e/ou sexual com uma outra pessoa, que possua algum grau de estabilidade. Podem ser considerados relacionamentos de casal os namoros,

Endereços para correspondência:

${ }^{1}$ João Fernando Rech Wachelke. R. Octavio Lebarbenchon, 69 - Santa Mônica - Florianópolis-SC - 88037-290

E-mail: wachelke@yahoo.com.br - Tel.: (48) 233-3785 / (48) 9902-3122

2 Roberto Moraes Cruz. R. Cap. Romualdo de Barros, 776, ap. 103C - Florianópolis-SC - 88040-001

E-mail: rcruz@cfh.ufsc.br. - Tel.: (48) 331-9904 / Fax: (48) 331-9984 
noivados e casamentos, bem como relacionamentos mais informais como casamentos não-oficiais ou namoros sem compromisso (o que se chama de "ficar", especialmente no meio jovem).

Para Arriaga (2001), que faz referência à teoria da interdependência de Kelley e Thibaut, a satisfação que um indivíduo possui acerca de um relacionamento está ligada a uma avaliação individual da positividade que existe no relacionamento. Nesses casos, uma pessoa pode ser considerada satisfeita com seu relacionamento quando os produtos de uma relação superam o que é esperado dessa relação. De acordo com Arriaga, um nível de satisfação alto e estável está associado à existência prolongada de um relacionamento no decorrer do tempo. A satisfação com o relacionamento, por sua vez, aumenta quando há maior envolvimento emocional das pessoas que se relacionam, bem como maior confiança, abertura para comunicação e interdependência (Sanderson \& Cantor, 1997; Rubin, Hill, Peplau \& Dunkel-Schetter, 1980; Hendrick, 1981). Féres-Carneiro (1997) descobriu que homens e mulheres heterossexuais valorizam a fidelidade, a integridade, o carinho e a paixão quando estão escolhendo parceiros; a satisfação com um relacionamento poderia, de acordo com nossa perspectiva, corresponder a avaliações positivas de algumas dessas dimensões, o que vem ao encontro das pesquisas citadas a respeito da relação entre envolvimento emocional, intimidade e satisfação com o relacionamento.

Rusbult (1983) trata a satisfação em relacionamento de forma semelhante a Arriaga (2001), ao propor um modelo teórico para o envolvimento romântico que se assemelha a um esquema econômico de perdas e ganhos. Para a autora, a satisfação corresponde às recompensas percebidas no relacionamento menos os custos despedidos no mesmo, num contexto de comparação. Dessa forma, uma pessoa com baixa exigência, que derive mais ganhos que perdas pessoais de seu relacionamento, pode ser considerada satisfeita com sua relação amorosa.

Por outro lado, qualidade de relacionamento é um construto mais amplo que a satisfação. Trata-se de uma avaliação global subjetiva, emitida por parte de um indivíduo, sobre seu relacionamento de casal (Fincham \& Bradbury, 1987), ou seja, uma avaliação da relação como boa, ruim, razoável, e assim por diante. De acordo com o modelo teórico proposto por Fletcher, Simpson e Thomas (2000), a qualidade de relacionamento pode ser predita com base em outros construtos de ordem hierárquica inferior, como, por exemplo, o compromisso com o relacionamento, a intimidade e a própria satisfação. Nesse sentido, a qualidade de relacionamento percebida não corresponde à satisfação, embora esta possua influência sobre aquela. A satisfação com o relacionamento é entendida como um componente do construto mais abrangente qualidade de relacionamento, que permite aos indivíduos julgarem com certa independência aspectos que refletem uma avaliação global da relação. Portanto, um relacionamento pode ser percebido por um indivíduo como bom (qualidade de relacionamento) mesmo que ele esteja pouco satisfeito com ele; afinal, outras dimensões da qualidade também têm peso na determinação da avaliação da relação. É relevante notar que, de acordo com esse modelo, os próprios construtos de ordem inferior também possuem componentes. Trata-se dos itens das subescalas específicas de construtos constituintes da qualidade de relacionamento.

A satisfação com relacionamento de casal é medida de muitas formas no cenário das pesquisas sobre a vida conjugal e outros relacionamentos. Seguem-se alguns exemplos, incluindo alguns dos mais difundidos instrumentos entre os estudiosos da área. Rusbult (1983) sugeriu uma escala de satisfação composta por três itens gerais no formato Likert de 9 pontos. A escala mede a satisfação geral de um relacionamento com itens que prezam por uma avaliação global, não ligada a comportamentos ou avaliações de aspectos específicos, mas, sim, ao relacionamento como um todo. Semelhante à medida de Rusbult, no que se refere à quantidade, formato e natureza dos itens, é a Kansas Marital Satisfaction Scale (Schumm et al., 1986), também composta por três itens no formato escala de 9 pontos. Essa escala requer que o sujeito analise o quão verdadeiras são algumas avaliações gerais do relacionamento. A subescala Global Satisfaction da Relationship Rating Form (RRF) de Davis (1996) é maior, composta por quatro subescalas com três itens cada: sucesso, apreciação, estima e reciprocidade. Os itens têm formato do tipo Likert de 7 a 9 pontos. Já a dimensão satisfação da Dyadic Adjustment Scale (DAS) (Spanier, 1976) possui itens que versam sobre o futuro, avaliação, harmonia e outros aspectos relacionados do relacionamento (Vinokur, Price \& Caplan, 1996). Diferentemente das escalas já citadas, a DAS trata tanto de avaliações gerais quanto de avaliações de aspectos distintos do relacionamento, visando chegar à satisfação geral. Também Simpson (1987) mediu a satisfação com um parceiro de relacionamento por meio de 11 itens sobre no formato de escalas Likert de 7 pontos, incluindo atração física, recursos financeiros, habilidade de prover apoio emocional, confiança, similaridade de atitudes e valores, compreensão, similaridades de interesses e atividades, atração sexual, proximidade e outros. Esses itens formaram uma escala de 11 componentes, com $\alpha$ de Cronbach 0,85. Além desses, existem muitos outros instrumentos, dentre testes e escalas, destinados a medir satisfação e qualidade de relacionamento, e mesmo medidas com um único item (Blum \& Mahrabian, 1999; Hendrick, 1988; Nakano et al., 2002; Sanderson \& Cantor, 1997). 
Embora os esforços para mensurar a qualidade de relacionamento e a satisfação sejam numerosos na literatura, o mesmo não se aplica aos componentes da satisfação em relacionamento de casal. Exceção a essa afirmativa é o estudo de Hernandez \& Oliveira (2003), em que há esforço de identificação de preditores para a satisfação com o relacionamento. Os autores, por meio de análises de regressão múltipla, identificaram a intimidade comunicativa - sentimento de ser compreendido pelo companheiro, sendo capaz de efetivamente se comunicar com ele e confiar nele - como principal prognosticador da satisfação nesse contexto, seguida pela excitação física. Esses resultados podem nortear a identificação de componentes da satisfação com o relacionamento, ou seja, "satisfações" referentes a aspectos localizados do relacionamento que juntos formem uma satisfação global da relação.

O interesse deste estudo está voltado principalmente para as ramificações do construto satisfação com o relacionamento de casal. Parte-se do pressuposto básico de que o relacionamento pode ser considerado separadamente em seus diversos aspectos, cada um dos quais pode satisfazer ou não o sujeito, que faz uma confrontação global dessas percepções para chegar à satisfação com o relacionamento de casal, em conformidade com o modelo teórico sugerido por Fletcher, Simpson \& Thomas (2000), modelo esse que obteve resultados expressivos em uma análise fatorial confirmatória. Ao lado disso, a própria satisfação interage com outros fatores para dar origem ao fator de ordem maior qualidade de relacionamento. Nesse sentido, o enfoque dado neste estudo corresponde a uma abordagem molecular da qualidade de relacionamento de casal; deseja-se caracterizar a magnitude de aspectos específicos da satisfação com a relação de casal, diferentemente do que se observa na grande maioria dos estudos sobre relacionamentos, cujos autores voltam-se sobretudo para a satisfação e percepção sobre a qualidade.

O objetivo deste estudo é descrever o processo de desenvolvimento e validação de uma escala bifatorial de satisfação em relacionamento de casal. Não foi do interesse dos autores criar uma medida da satisfação global no relacionamento, mas sim de componentes desse construto, no caso, a satisfação com a atração física e contato físico do relacionamento e outro referente à satisfação com as afinidades e semelhanças do casal.

\section{Método}

\section{Desenvolvimento da Escala - Etapa I}

Tendo em vista o objetivo final de criação de uma escala psicométrica de alguns fatores constituintes da satisfação em relacionamento de casal, o desenvolvimento do instrumento iniciou-se com um levantamento de indicadores de satisfação em relacionamentos de casal. Esses indicadores, por sua vez, norteariam o conteúdo dos itens que seriam submetidos em etapas posteriores a uma análise fatorial para determinação da dimensionalidade da escala.

\section{Participantes}

Participaram desta etapa de levantamento inicial do conteúdo da escala 10 participantes de ambos os sexos (5 homens e 5 mulheres, com idade variando de 17 a 25 anos), todos namorando à época do inquérito. Posteriormente, 44 participantes, todos eles estudantes de psicologia de uma universidade federal (o grupo de participantes, com idade média de 22 anos e 9 meses desvio padrão 4,69 -, foi composto por 27 mulheres e 17 homens) responderam a um questionário destinado a selecionar o conteúdo dos itens.

\section{Elaboração de Itens}

O questionário inicial foi constituído por uma pergunta aberta: "o que você considera importante para que um namoro seja satisfatório"? Os 10 indivíduos que participaram dessa etapa da pesquisa tiveram liberdade para mencionar tudo aquilo que considerassem pertinente. Após categorização das respostas obtidas, foram identificados quatro indicadores que poderiam constituir fatores da escala, uma vez que expressavam satisfação com aspectos distintos do relacionamento: satisfação em relação às afinidades de idéias e comportamentos entre os companheiros de relacionamento, satisfação em relação à atração física, satisfação com relação ao envolvimento emocional existente no relacionamento e satisfação com o tempo disponivel para o relacionamento.

Foram elaborados 8 itens no formato de escala Likert de 10 pontos (variando do extremo 1, discordo fortemente, a 10, concordo fortemente), relacionados semanticamente a cada um dos indicadores. Esses itens constituíram um questionário que foi respondido por outros 44 participantes. Tomando-se como pressuposto que os itens estariam medindo os indicadores derivados do levantamento inicial com os 10 participantes, foi calculada a média aritmética para cada indicador. O indicador atração física foi calculado pela média de 2 itens (exemplo: "considero meu namorado(a) fisicamente atraente"); o indicador afinidades de idéias e comportamentos foi formado por outros 2 itens (exemplo: "meu namorado(a) e eu gostamos de participar de atividades similares"). O indicador envolvimento emocional foi determinado como a média de 3 itens (exemplo: "Sou uma pessoa apaixonada por meu namorado(a)"). Tempo disponível foi mensurado como um único item ("considero o tempo que tenho disponível para meu relacionamento suficiente"). Também havia um item destinado a obter uma medida da satisfação global dos 
participantes com seus relacionamentos ("estou satisfeito com meu relacionamento com meu/minha namorado(a)"). Foram feitas correlações entre as medidas dos quatro indicadores e a medida de satisfação geral. O indicador tempo disponível obteve correlação inferior a 0,30 e foi desconsiderado no que diz respeito ao desenvolvimento posterior da escala. $\mathrm{O}$ indicador envolvimento emocional obteve a maior correlação $(0,78)$, seguido por afinidades de idéias e comportamentos $(0,52)$ e atração física $(0,36)$ (Faggiani, Andrade, Steffen, Wachelke \& Cruz, 2003).

Após essa filtragem inicial de conteúdo dos itens, foram construídos itens relacionados semanticamente aos indicadores atração física e afinidades de idéias e comportamentos entre as pessoas que se relacionam. Embora o indicador envolvimento emocional tivesse obtido maior correlação com a satisfação global, durante a elaboração de itens ele foi considerado vago, tendo em vista que a ligação do significado do indicador ao dos itens supostamente vinculados a ele não parecia tão clara para os autores e para alguns participantes que forneceram feedback após responderem o instrumento, em comparação com os outros dois indicadores. Além disso, a existência de afetos e emoções positivas que está implícita no entendimento do que viria a ser o indicador envolvimento emocional pode estar associada à satisfação com outras dimensões do relacionamento, como as próprias afinidades ou atração física. Nesse sentido, envolvimento emocional, enquanto um indicador, pode descaracterizar-se. Por esse motivo, foi decidido que seriam construídos itens com conteúdo voltado para os dois outros indicadores que apresentaram correlações satisfatórias.

\section{Desenvolvimento da Escala - Etapa II}

\section{Participantes}

Uma vez que a população-alvo da escala constitui-se de quaisquer pessoas que participem de um relacionamento de casal, buscou-se conseguir um grupo de participantes para validação da escala que fosse o mais diversificado possível. Responderam ao questionário que continha a versão final da escala 364 indivíduos que participavam de algum relacionamento de casal à época do inquérito, cujas idades variaram amplamente (de 13 a 77 anos), com média de idade de 25 anos e 11 meses (desvio padrão = 9,76 anos). 60,4\% deles foram do sexo feminino. Do total de participantes, 20,6\% participavam de relacionamento de casal há 6 meses ou menos; $24,5 \%$ deles possuía relacionamento com duração de 7 meses a 2 anos; $28,3 \%$ tinha esse relacionamento com duração de 2 a 5 anos e $26,6 \%$ possuía relacionamento há mais de 5 anos. 228 participantes $(62,6 \%)$ declararam que seus relacionamentos eram ótimos (ou seja, estavam satisfeitos com seus relacionamentos quando responderam ao questionário) enquanto o restante afirmou que seus relacionamentos poderiam ser melhores (declarando-se, então, insatisfeitos em maior ou menor grau, de forma geral).

\section{Elaboração de itens - versão final}

Para constituir a versão final da escala (Escala Fatorial da Satisfação em Relacionamento de Casal EFS-RC), foram elaborados 9 itens no formato escala de Likert de 5 pontos, em que os participantes deveriam responder o grau de concordância que eles tinham em relação às sentenças apresentadas. Todos os itens foram sentenças que diziam respeito a algum aspecto do relacionamento de casal do sujeito à época do inquérito (exemplos: "considero meu companheiro(a) bonito(a), "meu companheiro(a) e eu temos interesses e expectativas compatíveis em relação a nosso futuro profissional"). Além disso, estavam presentes no questionário perguntas de caracterização de gênero, idade, tempo de relacionamento, e satisfação com o relacionamento de forma geral (esta como um item de resposta fechada em que os participantes deveriam assinalar se julgavam seu relacionamento como ótimo ou passível de melhoras).

\section{Procedimento e análise}

A coleta de dados ocorreu em lugares públicos das cidades de Florianópolis e Porto Alegre, como praças de alimentação de shoppings, terminais de ônibus urbanos, campi universitários, e assim por diante. A aplicação dos questionários deu-se de forma individual. Ao se constatar que um sujeito estava sentado e desocupado momentaneamente, os aplicadores aproximavam-se e afirmavam estar realizando uma pesquisa da área de psicologia sobre relacionamentos de casal. Em seguida, perguntavam ao potencial sujeito de pesquisa se ele estava envolvido com algum relacionamento de casal. Em caso de resposta afirmativa, os aplicadores perguntavam a ele sobre a possibilidade e desejo de participar da pesquisa. Durante todo o processo, os pesquisadores e os assistentes de pesquisa treinados para a coleta de dados mostraram-se disponíveis para responder quaisquer dúvidas que assolassem os participantes, assegurando-se de que eles tinham uma compreensão adequada dos itens e da forma de resposta do questionário.

Para validação da escala foram realizadas análise dos componentes principais, análise fatorial exploratória com rotação varimax (visando-se à obtenção de fatores com ortogonalidade entre si) e cálculos de confiabilidade. O software SPSS v. 11 foi utilizado em todas as análises. 


\section{Resultados}

Primeiramente foi realizada a análise dos componentes principais, para verificar a adequação dos dados à análise fatorial e à quantidade de fatores. $\mathrm{O}$ KMO teve o valor de 0,80 , e o teste de esfericidade de Bartlett foi significativo no nível de 0,0001\%. O gráfico de sedimentação (Figura 1) indicou 2 fatores com eigenvalues superiores a 1, explicando no total 51,5\% da variância dos dados. Optou-se pela extração de 2 fatores.

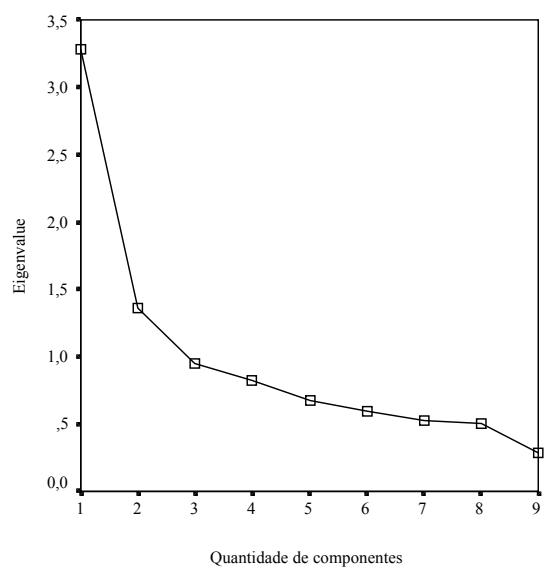

Figura 1 - Gráfico de sedimentação dos eigenvalues
Procedeu-se à realização da extração dos fatores principais (principal axis factoring) com rotação varimax e cálculos de confiabilidade. A rotação varimax foi escolhida, pois foi do interesse dos autores obter fatores com a maior ortogonalidade possível, uma vez que o objetivo principal é medir componentes do construto satisfação com relacionamento de casal com relativa independência entre si. Nenhum dos itens apresentou carga fatorial menor que 0,30, sendo todos os nove mantidos na composição da escala.

$\mathrm{Na}$ Tabela 1 estão apresentadas as cargas fatoriais maiores que 0,30 e comunalidades dos itens. Os fatores apresentaram correlação entre si de 0,36 , significativa no nível de $0,01 \%$. Os dois fatores encontrados receberam a seguinte nomeação: Satisfação com Atração Física e Sexualidade - SAFS (Fator 1); e Satisfação com Afinidades de Interesses e Comportamentos - SAIC (Fator 2).

O fator SAFS, composto por 5 itens $(2,7$ reverso, 4, 9 e 8), diz respeito à satisfação dos participantes em relação à aparência do sujeito e às situações de contato físico entre os companheiros. Trata-se de uma medida da satisfação com os aspectos mais corporais do relacionamento. Por outro lado, o fator SAIC, formado por 4 itens (1, 6, 3 e 5 reverso), está ligado à compatibilidade das pessoas que se relacionam, abrangendo suas idéias, preferências e ações.

Tabela 1 - Cargas fatoriais e comunalidade dos itens para análise fatorial exploratória

Fatores

2. Considero meu companheiro(a) bonito.

$\begin{array}{ccc}1 & 2 & h^{2} \\ 0,830 & 0,556\end{array}$

7. (reverso). A aparência de meu companheiro(a) NÃO é ideal para mim.

0,751

0,543

4. Meu companheiro é fisicamente atraente para mim.

0,658

0,377

9. As situações de contato físico entre meu companheiro(a) e eu são muito prazerosas.

0,546

0,334

8. Sinto muita vontade de engajar-me em situações de contato físico com meu companheiro(a).

1. Meu companheiro(a) e eu temos muitas idéias e interesses em comum.

0,334

6. Meu companheiro(a) e eu gostamos de participar de atividades similares.

0,272

3. Meu companheiro(a) e eu temos interesses e expectativas compatíveis em relação a nosso futuro profissional.

5. (reverso). Reprovo muitas atitudes de meu companheiro(a).

Método de extração: Fatoração do Eixo Principal. Método de rotação: Varimax com normalização Kaiser

A Tabela 2 apresenta a quantidade de itens, a variância explicada por fator e os índices de confiabilidade do tipo alfa de Cronbach de cada um deles, na fatoração do eixo principal. O primeiro fator explicou $24,85 \%$ da variância e o segundo, $14,03 \%$. O fator SAFS apresentou alfa de Cronbach com o valor de 0,76 - considerado satisfatório por ser maior que 0,70 (Cronbach, 1951; Nunnally, 1978). Contudo, o fator SAIC teve alfa de apenas 0,61, indicador de confiabilidade apenas moderada, o que sugere a necessidade de aprimorar em estudos posteriores esse fator para que a escala possa alcançar bons índices de fidedignidade, que podem ser obtidos pela adição de novos itens ou pela reformulação de itens já utilizados. De qualquer forma, de acordo com Nunnally (1978) é permissível a utilização de escalas com coeficientes alfa maiores que 0,60. 
Tabela 2 - Alfa de Cronbach, variância explicada e quantidade de itens relativos aos fatores Satisfação com Atração Física e Sexualidade (SAFS - Fator 1) e Satisfação com Afinidades de Interesses e Comportamentos (SAIC - Fator 2)

\begin{tabular}{ccc}
\hline & FAFS (1) & Fatores \\
\hline Alfa de Cronbach & 0,76 & SAIC (2) \\
Variância explicada & 24,85 & 0,61 \\
Quantidade de itens & 5 & 14,03 \\
\hline
\end{tabular}

A Tabela 3 apresenta as médias, o desvio padrão e a variância dos dois fatores para a população em geral. Os 364 participantes que responderam à escala tiveram médias mais altas de satisfação com os aspectos corporais do relacionamento que com os aspectos de compatibilidade; sua média no SAFS foi 4,59 e no SAIC foi 3,79. Além disso, o SAIC possui maior desvio padrão $(0,83)$ em relação ao $\operatorname{SAFS}(0,62)$, indicando maior variabilidade dos resultados.

Considerando que os itens da escala têm formato Likert de 5 pontos, sendo 3 o ponto médio, os resultados médios de ambas as subescalas sugerem que os participantes tendem mais à satisfação, no que diz respeito às duas dimensões mensuradas de seus relacionamentos, do que à insatisfação. Em média, o valor da subescala SAFS, próximo de 5, sugere que os participantes estão fortemente satisfeitos com o componente físico de seus relacionamentos. Embora o valor médio do fator SAIC também esteja situado entre os valores considerados indicadores de satisfação na escala (entre 3 e 5), ele está mais próximo do ponto médio que do extremo $5 \mathrm{e}$, portanto, deve ser interpretado como uma satisfação de menor grau. Parece ser correto dizer que a satisfação em relação às afinidades entre os companheiros de relacionamento é de modo geral menor e mais variável entre os diversos participantes, enquanto a satisfação com a sexualidade e aparência dos companheiros é maior e apresenta medidas mais parecidas entre si.

Tabela 3 - Distribuição das médias, desvio padrão e variâncias dos fatores Satisfação com Atração Física e Sexualidade (SAFS - Fator 1) e Satisfação com Afinidades de Interesses e Comportamentos (SAIC - Fator 2)

\begin{tabular}{ccccc}
\hline Fatores & $\mathrm{N}$ & Média & Desvio padrão & Variância \\
\hline SAFS (1) & 364 & 4,59 & 0,62 & 0,39 \\
SAIC (2) & 364 & 3,79 & 0,83 & 0,69 \\
\hline
\end{tabular}

Para verificar se a escala podia diferenciar com sucesso indivíduos satisfeitos com os aspectos referentes aos dois fatores, foi utilizado um item em que os participantes avaliaram seus relacionamentos de forma geral, em um dos seguintes campos: "O relacionamento está ótimo no momento atual" e "o relacionamento poderia ser melhor que é no momento atual". Os participantes que assinalaram a primeira resposta foram considerados satisfeitos com o relacionamento de forma geral, enquanto aqueles que assinalaram a segunda foram considerados pouco satisfeitos com seu relacionamento.

Como mostram os dados da Tabela 4, tanto a média do SAFS quanto a do SAIC apresentaram diferenças estatisticamente significativas em relação aos dois grupos com diferentes níveis de satisfação com o relacionamento, no nível de 99,9\%. Os valores médios dos grupos satisfeitos foram mais altos que os dos grupos pouco satisfeitos, para ambos os fatores. Embora no SAIC o grau de satisfação média tenha sido menor que no SAFS, foi verificada maior diferença (cerca de 0,8 ) entre satisfeitos e pouco satisfeitos nas médias do fator de afinidades. Nesse primeiro estudo, a subescala SAFS mostrou diferenciação mais sutil entre grupos diferentes, enquanto a escala do fator SAIC permitiu maior distinção entre as duas condições de satisfação com o relacionamento de casal.

Tabela 4 - Distribuição das médias e desvio padrão das subescalas dos fatores SAFS e SAIC de grupos de participantes que se declararam satisfeitos e pouco satisfeitos com seus relacionamentos

\begin{tabular}{ccccc}
\hline & Satisfação geral com o relacionamento & $\mathrm{N}$ & Média & Desvio padrão \\
\hline \multirow{2}{*}{ SAFS (1) } & Satisfeito & 228 & 4,69 & 0,51 \\
& Pouco satisfeito & 136 & 4,42 & 0,75 \\
\hline \multirow{2}{*}{ SAIC (2) } & Satisfeito & 228 & 4,09 & 0,65 \\
& Pouco satisfeito & 136 & 3,29 & 0,85 \\
\hline
\end{tabular}

Nota. Teste $t$ para amostras independentes das médias do SAFS: $t=3,838, \mathrm{p}<0,001(\mathrm{gl}=209)$. Teste $t$ para amostras independentes das médias do SAIC: $t=9,344, \mathrm{p}<0,001$ ( $\mathrm{gl}=229)$. 


\section{Discussão}

Os resultados da análise fatorial apontam para uma escala composta por dois fatores, Satisfação com Atração Física e Sexualidade (SAFS) e Satisfação com Afinidades de Interesses e Comportamentos (SAIC), constituídos por 5 e 4 itens, respectivamente, com boas cargas fatoriais. A validade de conteúdo da escala foi determinada nas primeiras etapas de pesquisa, em que foram ouvidos participantes sobre o que é considerado importante para um relacionamento e foram efetuadas correlações entre os indicadores criados e uma medida da satisfação global com o relacionamento. A validade de critério pode ser considerada contemplada, uma vez que participantes mais satisfeitos e menos satisfeitos com seus relacionamentos obtiveram escores com médias com diferenças estatisticamente significativas para ambas as subescalas.

A escala sustenta-se no modelo de qualidade de relacionamento de Fletcher, Simpson e Thomas (2000), segundo o qual a qualidade do relacionamento é composta por fatores de ordem hierárquica inferior, com relativa independência entre si, como por exemplo, a satisfação com o relacionamento. Por sua vez, a satisfação também possui componentes com uma certa separação; são esses componentes que se buscou medir com a escala descrita neste estudo. Dois desses fatores constituintes da satisfação com relacionamento de casal podem ser mensurados pela escala. É importante ressaltar que não necessariamente esses fatores são os mais importantes componentes da satisfação, ou mesmo bons preditores da satisfação com relacionamento de casal; tais constatações não podem ser feitas no âmbito deste estudo. O objetivo desta pesquisa foi desenvolver uma medida para alguns componentes fatoriais da satisfação com relacionamento de casal, objetivo este que pode ser considerado cumprido com a criação da escala bifatorial descrita.

Hernandez e Oliveira (2003) verificaram que a intimidade comunicativa e a excitação física são bons preditores da satisfação com o relacionamento. Parecenos correto dizer que excitação física e o fator SAFS podem ser considerados equivalentes, indicando a presença de suporte teórico para a escala. Contudo, é aconselhável elaborar uma medida psicológica para caracterizar também a intimidade e o envolvimento emocional entre as pessoas que se relacionam, dado que outros autores afirmam que esses fatores são fortes preditores de satisfação (Hendrick, 1981; Rubin, Hill, Peplau \& DunkelSchetter, 1980; Sanderson \& Cantor, 1997). Em virtude da semelhança das definições dos dois fatores, não se descarta também a hipótese de que eles se sobreponham, isto é, que estejam, em parte, medindo o mesmo construto. É possível que exista forte intersecção, também, entre o que se chama de intimidade comunicativa e as afinidades entre companheiros. De todo modo, somente com a acumulação de dados empíricos será possível dispor de noções mais precisas acerca dessa questão.

É recomendável que as investigações dos componentes da satisfação com relacionamento sejam ampliadas, de forma que gradativamente se possa chegar a uma sistematização mais completa desse construto. Uma vez que boas medidas de fatores da satisfação sejam desenvolvidas, é possível descobrir a contribuição precisa de cada um deles para a satisfação geral com o relacionamento de casal, integrando assim diversos níveis avaliativos da qualidade do relacionamento, por meio de procedimentos estatísticos como a regressão múltipla.

Conclui-se que a escala apresentada pode ser utilizada em pesquisas e diagnósticos de relacionamentos, principalmente como acessório para outras técnicas, na forma de um instrumento de mensuração breve e de fácil aplicação. No entanto, se há uma limitação da escala, está situada em sua confiabilidade, principalmente no que diz respeito ao fator de afinidades entre as pessoas que se relacionam, o que serve como indicativo da necessidade de futuramente aperfeiçoar a escala. Além disso, a escala não esgota os fatores componentes da satisfação com o relacionamento de casal, apenas possibilita medir dois deles, percebidos como presentes nas verbalizações dos participantes que auxiliaram a construir a escala por meio de feedback e também nos relacionamentos em geral, com maior ou menor importância. Por essas razões, parece-nos mais aconselhável aliar o emprego da escala a alguma outra técnica, como por exemplo uma entrevista de exploração, de forma a situar a medida em um locus de controle mais específico. Além disso, é importante considerar a necessidade de replicar o estudo com outras amostras e verificar a estabilidade e adequação da estrutura encontrada para outros públicos e outras regiões do país, ampliando o esforço de validação da escala.

\section{Referências}

Arriaga, X. B. (2001). The ups and downs of dating: fluctuations in satisfaction in newly formed romantic relationships. Journal of Personality \& Social Psychology, 80(5), 754-765.

Blum, J. S. \& Mahrabian, A. (1999). Personality and temperament correlates of marital satisfaction. Journal of Personality, 67, 93-125.

Cronbach, L. J. (1951). Coefficient alpha and the internal structure of tests. Psychometrika, 16(3), 297-334.

Davis, K. E. (1996). The Relationship Rating Form (RRF): A measure of the characteristics of romantic relationships and friendships. Manuscrito não publicado. 
<www.cla.sc.edu/PSYC/faculty/daviske/LoveFrien dsMeasure.pdf $>$

Faggiani, R. B., Andrade, A. L. de, Steffen, F. V., Wachelke, J. F. R. \& Cruz, R. M. (2003). Desenvolvimento inicial de uma escala de satisfação em relacionamento de namoro. I Congresso Brasileiro de Avaliação Psicológica. Livro de Resumos. (p. 146). Campinas.

Féres-Carneiro, T. (1997). A escolha amorosa e interação conjugal na heterossexualidade e na homossexualidade. Psicologia Reflexão e Crítica, 10(2), 351-368.

Ferreira, A. B. H. (1988). Dicionário Aurélio Básico da Lingua Portuguesa. Rio de Janeiro: Nova Fronteira.

Fincham, F. D. \& Bradbury, T. N. (1987). The assessment of marital quality: A re-evaluation. Journal of Marriage and the Family, 49, 797-810.

Fletcher, G. J. O., Simpson, J. A. \& Thomas. G. (2000). The measurement of perceived relationship quality components: A confirmatory factor analytic approach. Personality and Social Psychology Bulletin, 26(3) 340-354.

Hendrick, S. S. (1981). Self-disclosure and marital satisfaction. Journal of Personality and Social Psychology, 40, 1150-1159.

Hendrick, S. S. (1988). A generic measure of relationship satisfaction. Journal of Marriage and the Family, 50, 93-98.

Hernandez, J. A. E. \& Oliveira, I. M. B. de. (2003). Os componentes do amor e a satisfação. Psicologia Ciência e Profissão, 23(1), 58-69.

$<$ http://www.pol.org.br/revistapcp/artigos/23_01/arti go08_1.htm>

Nakano, Y., Sigiura, M., Aoki, K., Hori, S., Oshima, M., Kitamura T. \& Furukawa, T. (2002). A Japanese version of the Quality of Relationship Inventory: Its reliability and validity among women with recurrent spontaneous abortion. Psychiatry and Clinical Neurosciences, 56, 527-532.
Nunnally, J. C. (1978). Psychometric Theory. New York: McGraw-Hill.

Rubin, Z., Hill, C. T., Peplau, L. A. \& Dunkel-Schetter, C. (1980). Self-disclosure in dating couples: Sex roles and the ethic of openness. Journal of Marriage and the Family, 42, 305-317.

Rusbult, C. E. (1983). A longitudinal test of the investment model: The development (and deterioration) of satisfaction and commitment in heterosexual involvements. Journal of Personality and Social Psychology, 45, 101-117.

Sanderson, C. A., Cantor, N. (1997). Creating satisfaction in steady dating relationships: The role of personal goals and situational affordances. Journal of Personality \& Social Psychology, 73(6), 1424-1433.

Schumm, W. R., Paff-Bergen, L. A., Hatch, R. C., Obiorah, F. C., Copeland, J. E., Meens, L. D. \& Bugaighis, M. A. (1986). Concurrent and discriminant validity of the Kansas Marital Satisfaction Scale. Journal of Marriage and the Family, 48, 381-388.

Simpson, J. A. (1987). The dissolution of romantic relationships: Factors involved in relationship stability and emotional distress. Journal of Personality and Social Psychology, 53, 683-692.

Spanier, G. B. (1976). Measuring dyadic adjustment: New scales for assessing the quality of marriage and similar dyads. Journal of Marriage and the Family, 38, 15-28.

Vinokur, A. D., Price, R. H. \& Caplan, R. D. (1996). Hard times and hurtful partners: How financial strain affects depression and relationship satisfaction of unemployed persons and their spouses. Journal of Personality and Social Psychology, 71(1), 166-179.

Enviado em marco de 2004 Reformulado em maio de 2004 Aprovado em maio de 2004

Sobre os autores:

João Fernando Rech Wachelke é acadêmico de Psicologia da Universidade Federal de Santa Catarina, integrante da equipe do Laboratório de Psicossociologia da Comunicação e da Cognição Social.

Alexsandro Luiz de Andrade é acadêmico de Psicologia da Universidade Federal de Santa Catarina.

Roberto Moraes Cruz é psicólogo, mestre em Educação e doutor em Engenharia de Produção, professor do curso de graduação e pós-graduação em Psicologia da Universidade Federal de Santa Catarina e coordenador do Laboratório de Psicologia do Trabalho e Ergonomia.

Robson Brino Faggiani é acadêmico de Psicologia da Universidade Federal de Santa Catarina, integrante da equipe do Laboratório de Psicologia Experimental.

Jean Carlos Natividade é acadêmico de Psicologia da Universidade Federal de Santa Catarina. 